\title{
Allogeneic Dendritic Cell-Myeloma Idiotype Vaccine
}

National Cancer Institute

\section{Source}

National Cancer Institute. Allogeneic Dendritic Cell-Myeloma Idiotype Vaccine. NCI

Thesaurus. Code C61434.

A cell-based vaccine composed of allogeneic dendritic cells pulsed ex-vivo with an autologous myeloma idiotype with potential antineoplastic activity. Upon administration, allogeneic dendritic cell-myeloma idiotype vaccine may stimulate the host immune system to mount a specific cytotoxic T-lymphocyte (CTL) response against myeloma cells, resulting in cell lysis. 